Revue d'histoire de l'Amérique française

REVUE D.HISTOIRE DE L'AMÉRIQUE FRANÇAISE

FALARDEAU, Jean-Charles et GARIGUE, Philippe, Léon Gérin et l'habitant de Saint-Justin. Les Presses de l'Université de Montréal, Montréal 1968. 179 p. \$2.85.

\title{
Pierre Savard
}

Volume 23, numéro 4, mars 1970

URI : https://id.erudit.org/iderudit/302938ar

DOI : https://doi.org/10.7202/302938ar

Aller au sommaire du numéro

Éditeur(s)

Institut d'histoire de l'Amérique française

ISSN

0035-2357 (imprimé)

1492-1383 (numérique)

Découvrir la revue

Citer ce compte rendu

Savard, P. (1970). Compte rendu de [FALARDEAU, Jean-Charles et GARIGUE, Philippe, Léon Gérin et l'habitant de Saint-Justin. Les Presses de l'Université de Montréal, Montréal 1968. 179 p. \$2.85.] Revue d'histoire de l'Amérique française, 23(4), 614-615. https://doi.org/10.7202/302938ar d'utilisation que vous pouvez consulter en ligne.

https://apropos.erudit.org/fr/usagers/politique-dutilisation/ 
Falardeau, Jean-Charles et Garigue, Philippe, Léon Gérin et l'habitant de Saint-Justin. Les Presses de l'Université de Montréal, Montréal 1968. 179 p. \$2.85.

Il est agréable et instructif pour l'historien et le public de se plonger de temps à autre dans des écrits d'époque qui restituent le passé vivant. Nous avons trop d'histoires par historiens interposés lesquels schématisent, abstraient, vident de sa substance quand ils ne dénaturent pas par la projection 
de leur problème présent (plus souvent au singulier qu'au pluriel) le passé qu'ils se donnent pour mission de faire comprendre. La monographie de Léon Gérin sur une famille d'habitants de Saint-Justin, dans le comté de Maskinongé, dans les années 1890 , est un classique de la sociographie québecoise. C'est une bonne action qu'on a fait en la reproduisant et en la rendant accessible à un large public. Aux côtés de l'histoire mystique dont nous ne sommes pas sortis et l'histoire scientifique qui n'intéresse souvent que les historiens frappés de ce mal, il y a encore de la place pour les témoins avec leurs servitudes et leurs grandeurs.

Le lecteur de Gérin découvre bien des aperçus riches et neufs, par exemple, sur la culture populaire de nos ancêtres de cette époque (apparition des dime novels, etc.). Il se fait rappeler l'importance de certains traits durables de la mentalité comme la faiblesse des rendements agricoles ou l'autorité limitée du père. Et il trouve des preuves de plus, s'il en faut, contre des lieux communs de l'historiographie et de l'éloquence traditionnelle (par exemple, la continuité de la propriété). Et, bien entendu, il doit garder à l'esprit tout au long de la lecture que Gérin dit décrire "un type prospère mais non exceptionnel" (p. 57). On sait que ce genre d'étude a tendance à privilégier l'exemplaire plutôt qu'à dégager le moyen.

Au texte de Gérin, on a fort justement ajouté une introduction à sa vie et à son œuvre par le sociologue Jean-Charles Falardeau. Les commentaires de Falardeau sur les insuffisances de la méthode de Le Play intéresseront peu l'historien. Par contre, celui-ci tirera beaucoup de l'analyse fine que Falardeau fait de Gérin: une voix dans le désert et un homme resté proche de l'idéologie de l'élite qui idéalise légèrement "le bon habitant". La bibliographie des travaux de Gérin et des études sur sa vie et son œuvre patiemment compilée par Falardeau nous rappelle comment ce genre de travail reste ardu au Québec où les grands instruments (bibliographies, catalogues, etc.) font souvent défaut.

On a eu la bonne idée d'ajouter à ce dossier de Saint-Justin et de Léon Gérin la monographie de la paroisse qu'a faite, au milieu des années 1950 , le sociologue Philippe Garigue. On y voit vivre une communauté "rurale" québecoise à la veille de la "révolution tranquille". Lecture tonifiante qui repose des élucubrations abstraites sur le Québec global, et rappelle que le $19 \mathrm{e}$ siècle est encore proche de nous.

Institut d'histoire

Pierre SAVARD

Université Laval 